International Journal of Applied Mathematical Research, 5(1) (2016) 29-38
International Journal of Applied Mathematical Research
SPC
Website: www.sciencepubco.com/index.php/IJAMR
doi: $10.14419 /$ ijamr.v5il.5581
Research paper

\title{
Conducting dusty fluid flow through a constriction in a porous medium
}

\author{
K. R. Madhura ${ }^{1}$ and Uma. M. S..$^{2 *}$ \\ ${ }^{1}$ Postgraduate department of Mathematics, The National Degree College, Jayanagar, Bangalore, Karnataka, India \\ ${ }^{2}$ Department of Physics, Jyothy Institute of Technology, Bangalore, Karnataka, India \\ *Corresponding author E-mail: umamsjit@gmail.com
}

\begin{abstract}
The flow of an unsteady incompressible electrically conducting fluid with uniform distribution of dust particles in a constricted channel has been studied. The medium is assumed to be porous in nature. The governing equations of motion are treated analytically and the expressions are obtained by using variable separable and Laplace transform techniques. The influence of the dust particles on the velocity distributions of the fluid are investigated for various cases and the results are illustrated by varying parameters like Hartmann number, deposition thickness on the walls of the cylinder and the permeability of the porous medium on the velocity of dust and fluid phase.
\end{abstract}

Keywords: Bessel function; Dusty fluid; Naviers Strokes equations; Porous media.

\section{Introduction}

Advances in mathematical modelling of fluids with more than single phase continue to be of crucial importance in many diversified problems like fluidization, purification of crude oil, centrifugal separation of matter from fluid, petroleum industry, dust in gas cooling systems, performance of solid fuel rocket nozzles and blood flow in capillaries and other suspended particles in seas and oceans.

A number of studies associated with flow of fluids embedded with particles have appeared in literature. Saffman [1] carried out pioneering work on the stability of a laminar flow of a dust gas in which the dust particles are uniformly distributed. Michael and Miller [2] have studied the motion of dusty gas with uniform distribution of the dust particles which occupied in the semi-infinite space above a rigid plane boundary. Sleep [3] discussed the modelling transient organic vapour transport in porous media with the dusty gas model. P. Mitra and P. Bhattacharyya [4] have studied the unsteady hydromagnetic laminar flow of a conducting dusty fluid [5] between two parallel plates started impulsively from rest. Chamkha $[6,7]$ has studied the unsteady flow of electrically conducting dusty gas in a channel due to an oscillating pressure gradient. Lokenath and Ghosh [8] have studied the unsteady hydromagnetic flows of dusty fluid between two oscillating plates. A magnetohydrodynamic convection flow of an electrically conducting heat generating fluid past a semi-infinite vertical porous plate with variable suction was studied by Nicholas $e t$ al. [9]. Dusty fluid flow in Frenet frame field system was studied by Bagewadi and Gireesha $[10,11]$ by applying differential geometry techniques. Further, fluid flow in a constricted channel is encountered in many practical problems like narrowing of pipeline network in drinking water distribution systems and sewage systems. Analysis of fluid flow in systems with constriction helps one to understand the mechanism of migration of suspended heavy organic particles towards the walls in oil-producing wells and pipelines. The Mathematical modelling of blood flow through constricted channels has drawn serious attention of researchers because of recent advances in understanding the effects of stenosis in blood flow and the optimal design of artificial organs [12, 13]. Bio-mathematicians like Pralhad et al. [14, 15], Ponalagusamy [16], Chaturani et al. [17, 18] and Mishra $[19,20]$ have studied the blood flow through a stenosized artery by using mathematical techniques. Recently, an analytical solution for the fluid flow through a narrowing system was derived $[21,22]$. It is of interest in this paper to derive solutions for an electrically conducting dusty fluid flowing in a channel which is constricted due to the deposition thickness on the wall and thus analyze the velocity distributions of the fluid and dust phase.

\section{Equations of Motion}

The Naviers - Stokes Equations for an incompressible conducting dusty fluid flow through a porous medium are [1]:

For fluid phase

$\nabla \cdot \vec{u}=0$

$\frac{\partial \vec{u}}{\partial t}=-\frac{1}{\rho} \nabla p+v \nabla^{2} \vec{u}+\frac{K N}{\rho}(\vec{v}-\vec{u})+\frac{1}{\rho}(\vec{J} \times \vec{B})-\frac{\mu \vec{u}}{\eta}$,

For dust phase,

$\nabla \cdot \vec{v}=0$

$$
\frac{\partial \vec{v}}{\partial t}=\frac{K}{m}(\vec{u}-\vec{v})
$$




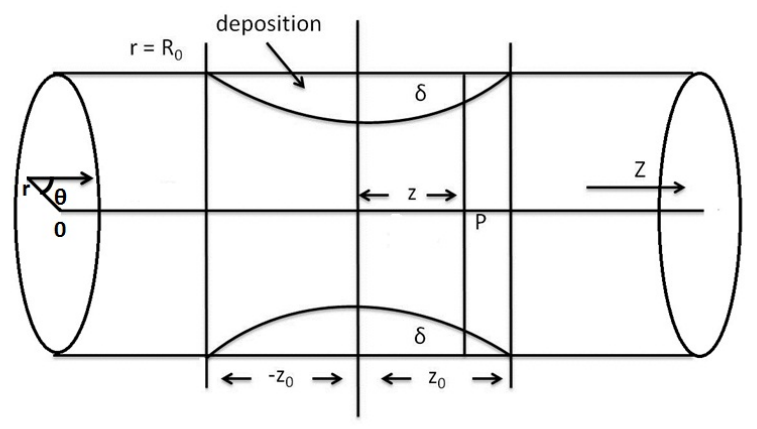

Figure 1: Geometry of the flow : $R_{0}=$ distance from the axis of the cylindrical boundary, $z=$ distance from $z=0$ to the point of calculation $P$.

We have following nomenclature:

$u$-velocity of the fluid phase, $v$-velocity of dust phase, $p-$ the fluid pressure, $\mathrm{m}-$ mass of the particle, $K=6 \pi \mu r-$ Stokes resistance coefficient with $\mu$ being the viscosity of the fluid and $r$ the radius of the spherical particle, $N$ - number density of the particle, $t-$ the time , $\rho$ - mass density of the particle, $v=\frac{\mu}{\rho}-$ the kinematic viscosity of the fluid, $\vec{J}$ and $\vec{B}$ are given by Maxwell's equations

$\nabla \times \vec{H}=4 \pi \vec{J}, \quad \nabla \times \vec{B}=0, \quad \nabla \times \vec{E}=0, \vec{J}=\sigma[\vec{E}+\vec{u} \times \vec{B}]$,

where $\vec{H}$ - magnetic field, $\vec{B}$ - magnetic flux, $\vec{J}$ - current density, $\vec{E}-$ electric field and $\sigma-$ the electrical condcutivity of the field. It is assumed that no external electric field is applied and the effect of the induced magnetic fields produced by the electrically conducting fluid is negligible. The magnetic field $\vec{J} \times \vec{B}$ simply reduces to $-\sigma B_{0}^{2} \vec{u}$, where $B_{0}$ is the intensity of the imposed transverse magnetic field.

\section{Formulation and Solution of the Problem}

Consider an unsteady laminar flow of an incompressible conducting dusty fluid with uniform distribution of dust particles through porous medium in a long circular cylinder in which the fluid is at rest initially. The flow is due to the influence of time dependent pressure gradients imposed along the axis of the cylinder. It is assumed that the dust particles are spherical in shape and uniform in size and number density of the dust particles is taken to be constant throughout the flow. Let $z$ be the direction of the axis of cylinder along which the flow takes place and let $r$ be the radial direction outward from the $z$ axis. Assumption is made that the channel is narrow due to the depositions of thickness $\delta$ on the wall of the cylinder. The elevation of thickness due to deposition is given by [21]

$R=R_{0}-\frac{\delta}{2}\left(1+\cos \frac{\pi z}{z_{0}}\right)$.

The axis of the channel is along $z$ axis and the velocity components of both fluid and dust particles are respectively given by:

$u_{r}=0 ; \quad u_{\theta}=0 ; \quad u_{z}=(r, \theta, t) \quad v_{r}=0 ; \quad v_{\theta}=0 ; \quad v_{z}=(r, \theta, t)$.

By virtue of 6 we can rewrite 2 and 4 as

$$
\begin{aligned}
& \frac{\partial u_{z}}{\partial t}=-\frac{1}{\rho} \frac{\partial p}{\partial z}+v\left(\frac{\partial^{2} u_{z}}{\partial r^{2}}+\frac{1}{r} \frac{\partial u_{z}}{\partial r}+\frac{1}{r^{2}} \frac{\partial^{2} u_{z}}{\partial \theta^{2}}\right)-\frac{\sigma B_{0}^{2} u_{z}}{\rho} \\
& +\frac{K N}{\rho}\left(v_{z}-u_{z}\right)-\frac{\mu u_{z}}{\eta} \\
& \frac{\partial v_{z}}{\partial t}=\frac{K}{m}\left(u_{z}-v_{z}\right) .
\end{aligned}
$$

The initial and the boundary conditions imposed on the system are

$t<0 ; \quad u(r, \theta, t)=0, \quad v(r, \theta, t)=0$,

$t>0 ; \quad u(r, \theta, t)=0, \quad v(r, \theta, t)=0 ;$ at $r=R, \theta= \pm \alpha$.

By introducing the following nondimensional quantities

$r^{*}=\frac{r}{R_{0}}, \quad R^{*}=\frac{R}{R_{0}}, \quad z^{*}=\frac{z}{R_{0}}, p^{*}=\frac{p R_{0}^{2}}{\rho v^{2}}, t^{*}=\frac{t v}{R_{0}^{2}}, \quad u=\frac{u_{z} R_{0}}{v}$, $v=\frac{v_{z} R_{0}}{v}, \delta^{*}=\frac{\delta}{R_{0}}, z_{0}^{*}=\frac{z_{0}}{R_{0}}$.

7 and 8 can be expressed as (after dropping the $*$ ),

$\frac{\partial u}{\partial t}=-\frac{\partial p}{\partial z}+\left(\frac{\partial^{2} u}{\partial r^{2}}+\frac{1}{r} \frac{\partial u}{\partial r}+\frac{1}{r^{2}} \frac{\partial^{2} u}{\partial \theta^{2}}\right)+\beta(v-u)-M^{2} u-P_{r} u$,

$\frac{\partial v}{\partial t}=\lambda(u-v)$,

where, $\beta=\frac{K N R_{0}^{2}}{\rho v}, \quad P_{r}=\frac{\mu R_{0}^{2}}{\eta v}, \quad M^{2}=\frac{\sigma B_{0}^{2} R_{0}^{2}}{\mu}, \quad \lambda=\frac{K R_{0}^{2}}{m v}$.

Accordingly, 5, 9 and 10 assume a nondimensional form

$R=1-\frac{\delta}{2}\left(1+\cos \frac{\pi z}{z_{0}}\right)$,

$t>0 ; u(r, \theta, t)=0, \quad v(r, \theta, t)=0 \quad$ at $\quad r=R$,

$$
u(r, \theta, t)=0, \quad v(r, \theta, t)=0 \quad \text { at } \quad \theta= \pm \alpha .
$$

Let $\wp(t)$ be the time dependent pressure gradient to be imposed on the system. So we can write

$-\frac{\partial p}{\partial z}=\wp(t)$.

Applying Laplace transform to 11 and 12 one arrives at

$\frac{d^{2} \bar{u}}{d r^{2}}+\frac{1}{r} \frac{d \bar{u}}{d r}+\frac{1}{r^{2}} \frac{d^{2} \bar{u}}{d \theta^{2}}-Q^{2} \bar{u}=-\phi(s)$,

where $Q^{2}=\left(s+M^{2}+\frac{\beta s}{s+\lambda}+P_{r}\right)$.

where $\bar{u}$ and $\bar{v}$ are the Laplace transforms defined by

$\bar{u}=\int_{0}^{\infty} e^{-s t} u d t$ and $\bar{v}=\int_{0}^{\infty} e^{-s t} v d t$,

and $\phi(s)$ is the Laplace Transform of $\wp(t)$.

After Laplace transform, the boundary conditions become

$\bar{u}(r, \theta, t)=0, \bar{v}(r, \theta, t)=0 \quad$ at $r=R, \theta= \pm \alpha$.

Let the solution of 13 be of the form

$\bar{u}(r, \theta)=w_{1}(r, \theta)+w_{2}(r)$.

Then, 13 becomes

$\frac{\partial^{2} w_{2}}{\partial r^{2}}+\frac{1}{r} \frac{\partial w_{2}}{\partial r}-Q^{2} w_{2}+\phi(s)=0$,

$\frac{\partial^{2} w_{1}}{\partial r^{2}}+\frac{1}{r} \frac{\partial w_{1}}{\partial r}+\frac{1}{r^{2}} \frac{\partial^{2} w_{1}}{\partial \theta^{2}}-Q^{2} w_{1}=0$.

The solutions of 15 are

$w_{2}(r)=\frac{\phi(s)}{Q^{2}}\left[1-\frac{I_{0}(Q r)}{I_{0}(Q R)}\right]$,

with $I_{0}(Q r)$ being the modified Bessel function of the first kind.

The solution for 16 is assumed to be

$w_{1}(r, \theta)=X(r) Y(\theta)$, 
with the conditions

$$
\begin{aligned}
w_{1}(0, \theta) & =\text { finite, } \quad w_{1}(r, \alpha)=-w_{2}(r), \\
w_{1}(r, \theta) & =0, \quad w_{1}(r,-\alpha)=-w_{2}(r) .
\end{aligned}
$$

One arrives at,

$w_{1}(r, \theta)=-w_{2}(r) \frac{\cos n \theta}{\cos n \alpha}$,

Thus,

$\bar{u}=\frac{\phi(s)}{Q^{2}}\left[1-\frac{I_{0}(Q r)}{I_{0}(Q R)}\right]\left[\frac{\cos n \alpha-\cos n \theta}{\cos n \alpha}\right]$,

$\bar{v}=\frac{\lambda \phi(s)}{(s+\lambda) Q^{2}}\left[1-\frac{I_{0}(Q r)}{I_{0}(Q R)}\right]\left[\frac{\cos n \alpha-\cos n \theta}{\cos n \alpha}\right]$.

\section{Particular Cases}

\subsection{Case-1: Impulsive motion}

In the case of impulsive motion, the pressure gradient is given by $\wp(t)=p_{0} \delta(t)$, where $\delta(t)$ is Dirac delta function and $p_{0}$ is a constant.

The equations 21 and 22 become

$\bar{u}=\frac{p_{0}}{Q^{2}}\left(\frac{I_{0}(Q R)-I_{0}(Q r)}{I_{0}(Q R)}\right)\left[\frac{\cos n \alpha-\cos n \theta}{\cos n \alpha}\right]$,

$\bar{v}=\frac{p_{0} \lambda}{(s+\lambda) Q^{2}}\left(\frac{I_{0}(Q R)-I_{0}(Q r)}{I_{0}(Q R)}\right)\left[\frac{\cos n \alpha-\cos n \theta}{\cos n \alpha}\right]$.

Laplace inverse of the of $\bar{u}$ and $\bar{v}$ give the velocity profiles

$$
\begin{aligned}
& u=\sum_{k_{m}=1}^{\infty} \frac{2 R p_{0}}{k_{m}}\left(\frac{I_{0}\left(k_{m}\right)-I_{0}\left(\frac{k_{m} r}{R}\right)}{I_{1}\left(k_{m}\right)}\right)\left[\frac{\cos n \alpha-\cos n \theta}{\cos n \alpha}\right] \\
& \times\left\{\frac{e^{y_{1} t}\left(y_{1}+\lambda\right)^{2}}{\left[\left(y_{1}+\lambda\right)^{2}+\lambda \beta\right]}+\frac{e^{y_{2} t}\left(y_{2}+\lambda\right)^{2}}{\left[\left(y_{2}+\lambda\right)^{2}+\lambda \beta\right]}\right\}, \\
& v=\sum_{k_{m}=1}^{\infty} \frac{2 R p_{0} \lambda}{k_{m}}\left(\frac{I_{0}\left(k_{m}\right)-I_{0}\left(\frac{k_{m} r}{R}\right)}{I_{1}\left(k_{m}\right)}\right)\left[\frac{\cos n \alpha-\cos n \theta}{\cos n \alpha}\right] \\
& \times\left\{\frac{e^{y_{1} t}\left(y_{1}+\lambda\right)}{\left[\left(y_{1}+\lambda\right)^{2}+\lambda \beta\right]}+\frac{e^{y_{2} t}\left(y_{2}+\lambda\right)}{\left[\left(y_{2}+\lambda\right)^{2}+\lambda \beta\right]}\right\},
\end{aligned}
$$

where $(m=1,2,3, \ldots)$ are the positive roots of $I_{0}(k)=0$.

Shear stress (Skin friction) The shear stress at the boundary at $r=R$ and $\theta= \pm \alpha$ for impulsive motion is given by

$$
\begin{aligned}
& D_{r, R}=-2 p_{0}\left\{\frac{e^{y_{1} t}\left(y_{1}+\lambda\right)^{2}}{\left[\left(y_{1}+\lambda\right)^{2}+\lambda \beta\right]}+\frac{e^{y_{2} t}\left(y_{2}+\lambda\right)^{2}}{\left[\left(y_{2}+\lambda\right)^{2}+\lambda \beta\right]}\right\} \\
& {\left[\frac{\cos n \alpha-\cos n \theta}{\cos n \alpha}\right]} \\
& D_{\theta, \alpha}=\sum_{k_{m}=1}^{\infty} \frac{2 R p_{0}}{k_{m}}\left\{\frac{e^{y_{1} t}\left(y_{1}+\lambda\right)^{2}}{\left[\left(y_{1}+\lambda\right)^{2}+\lambda \beta\right]}+\frac{e^{y_{2} t}\left(y_{2}+\lambda\right)^{2}}{\left[\left(y_{2}+\lambda\right)^{2}+\lambda \beta\right]}\right\} \\
& \left(\frac{I_{0}\left(k_{m}\right)-I_{0}\left(\frac{k_{m} r}{R}\right)}{I_{1}\left(k_{m}\right)}\right) \zeta \\
& D_{\theta,-\alpha}=-D_{\theta, \alpha}
\end{aligned}
$$

\subsection{Case-2 : Transition motion}

We have, $\wp(t)=p_{0} H(t) e^{-w t}$ where $H(t)$ is Heaviside step function. The solutions take the form

$u=\sum_{k_{m}=1}^{\infty} \frac{2 R p_{0}}{k_{m}}\left(\frac{I_{0}\left(k_{m}\right)-I_{0}\left(\frac{k_{m} r}{R}\right)}{I_{1}\left(k_{m}\right)}\right)\left[\frac{\cos n \alpha-\cos n \theta}{\cos n \alpha}\right]$

$\times\left\{\frac{e^{y_{1} t}\left(y_{1}+\lambda\right)^{2}}{\left[\left(y_{1}+\lambda\right)^{2}+\lambda \beta\right]\left(y_{1}+w\right)}+\frac{e^{y_{2} t}\left(y_{2}+\lambda\right)^{2}}{\left[\left(y_{2}+\lambda\right)^{2}+\lambda \beta\right]\left(y_{2}+w\right)}\right\}$

$+\frac{p_{0} e^{-w t}}{Q_{1}^{2}}\left[\frac{I_{0}\left(Q_{1} R\right)-I_{0}\left(Q_{1} r\right)}{I_{0}\left(Q_{1} R\right)}\right]\left[\frac{\cos n \alpha-\cos n \theta}{\cos n \alpha}\right]$,

$v=\sum_{k_{m}=1}^{\infty} \frac{2 \lambda R p_{0}}{k_{m}}\left(\frac{I_{0}\left(k_{m}\right)-I_{0}\left(\frac{k_{m} r}{R}\right)}{I_{1}\left(k_{m}\right)}\right)\left[\frac{\cos n \alpha-\cos n \theta}{\cos n \alpha}\right]$

$\times\left\{\frac{e^{y_{1} t}\left(y_{1}+\lambda\right)}{\left[\left(y_{1}+\lambda\right)^{2}+\lambda \beta\right]\left(y_{1}+w\right)}+\frac{e^{y_{2} t}\left(y_{2}+\lambda\right)}{\left[\left(y_{2}+\lambda\right)^{2}+\lambda \beta\right]\left(y_{2}+w\right)}\right\}$

$+\frac{p_{0} \lambda e^{-w t}}{Q_{1}^{2}(\lambda-w)}\left[\frac{I_{0}\left(Q_{1} R\right)-I_{0}\left(Q_{1} r\right)}{I_{0}\left(Q_{1} R\right)}\right]\left[\frac{\cos n \alpha-\cos n \theta}{\cos n \alpha}\right]$.

Shear stress (Skin friction) The shear stress at the boundary at $r=R$ and $\theta= \pm \alpha$

$D_{r, R}=-\frac{p_{0} e^{-w t}}{Q_{1}} \frac{I_{1}\left(Q_{1} R\right)}{I_{0}\left(Q_{1} R\right)}\left[\frac{\cos n \alpha-\cos n \theta}{\cos n \alpha}\right]-2 p_{0}\left[\frac{\cos n \alpha-\cos n \theta}{\cos n \alpha}\right]$

$\times\left\{\frac{e^{y_{1} t}\left(y_{1}+\lambda\right)^{2}}{\left[\left(y_{1}+\lambda\right)^{2}+\lambda \beta\right]\left(y_{1}+w\right)}+\frac{e^{y_{2} t}\left(y_{2}+\lambda\right)^{2}}{\left[\left(y_{2}+\lambda\right)^{2}+\lambda \beta\right]\left(y_{2}+w\right)}\right\}$

$D_{\theta, \alpha}=\sum_{k_{m}=1}^{\infty} \frac{2 R p_{0}}{k_{m}}\left(\frac{I_{0}\left(k_{m}\right)-I_{0}\left(\frac{k_{m} r}{R}\right)}{I_{1}\left(k_{m}\right)}\right) \zeta-\frac{p_{0} e^{-w t}}{Q_{1}}\left(\frac{I_{0}\left(k_{m}\right)-I_{0}\left(\frac{k_{m} r}{R}\right)}{I_{1}\left(k_{m}\right)}\right) \zeta$

$\times\left\{\frac{e^{y_{1} t}\left(y_{1}+\lambda\right)^{2}}{\left[\left(y_{1}+\lambda\right)^{2}+\lambda \beta\right]\left(y_{1}+w\right)}+\frac{e^{y_{2} t}\left(y_{2}+\lambda\right)^{2}}{\left[\left(y_{2}+\lambda\right)^{2}+\lambda \beta\right]\left(y_{2}+w\right)}\right\}$

$D_{\theta,-\alpha}=-D_{\theta, \alpha}$

\subsection{Case-3 : Motion for a finite time}

In this case, we consider $\wp(t)=p_{0}[H(t)-H(t-T)]$. The solutions obtained in this case are

$$
\begin{aligned}
& u=\sum_{k_{m}=1}^{\infty} \frac{2 R p_{0}}{k_{m}}\left(\frac{I_{0}\left(k_{m}\right)-I_{0}\left(\frac{k_{m} r}{R}\right)}{I_{1}\left(k_{m}\right)}\right)\left[\frac{\cos n \alpha-\cos n \theta}{\cos n \alpha}\right] \\
& \times\left\{\frac{e^{y_{1} t}\left(y_{1}+\lambda\right)^{2}\left(1-e^{-y_{1} T}\right)}{y_{1}\left[\left(y_{1}+\lambda\right)^{2}+\lambda \beta\right]}+\frac{e^{y_{2} t}\left(y_{2}+\lambda\right)^{2}\left(1-e^{-y_{2} T}\right)}{y_{2}\left[\left(y_{2}+\lambda\right)^{2}+\lambda \beta\right]}\right\}, \\
& v=\sum_{k_{m}=1}^{\infty} \frac{2 R p_{0} \lambda}{k_{m}}\left(\frac{I_{0}\left(k_{m}\right)-I_{0}\left(\frac{k_{m} r}{R}\right)}{I_{1}\left(k_{m}\right)}\right)\left[\frac{\cos n \alpha-\cos n \theta}{\cos n \alpha}\right] \\
& \times\left\{\frac{e^{y_{1} t}\left(y_{1}+\lambda\right)\left(1-e^{-y_{1} T}\right)}{y_{1}\left[\left(y_{1}+\lambda\right)^{2}+\lambda \beta\right]}+\frac{e^{y_{2} t}\left(y_{2}+\lambda\right)\left(1-e^{-y_{2} T}\right)}{y_{2}\left[\left(y_{2}+\lambda\right)^{2}+\lambda \beta\right]}\right\} .
\end{aligned}
$$

Shear stress (Skin friction) The shear stress at the boundary at $r=R$ and $\theta= \pm \alpha$

$D_{r, R}=-2 p_{0}\left\{\frac{e^{y_{1} t}\left(y_{1}+\lambda\right)^{2}\left(1-e^{-y_{1} T}\right)}{\left[\left(y_{1}+\lambda\right)^{2}+\lambda \beta\right] y_{1}}+\frac{e^{y_{2} t}\left(y_{2}+\lambda\right)^{2}\left(1-e^{-y_{2} T}\right)}{\left[\left(y_{2}+\lambda\right)^{2}+\lambda \beta\right] y_{2}}\right\}$ $\left[\frac{\cos n \alpha-\cos n \theta}{\cos n \alpha}\right]$

$D_{\theta, \alpha}=\sum_{k_{m}=1}^{\infty} \frac{2 R p_{0}}{k_{m}}\left\{\frac{e^{y_{1} t}\left(y_{1}+\lambda\right)^{2}\left(1-e^{-y_{1} T}\right)}{\left[\left(y_{1}+\lambda\right)^{2}+\lambda \beta\right] y_{1}}+\frac{e^{y_{2} t}\left(y_{2}+\lambda\right)^{2}\left(1-e^{-y_{2} T}\right)}{\left[\left(y_{2}+\lambda\right)^{2}+\lambda \beta\right] y_{2}}\right\}$ $\times\left(\frac{I_{0}\left(k_{m}\right)-I_{0}\left(\frac{k_{m} r}{R}\right)}{I_{1}\left(k_{m}\right)}\right) \zeta$

$D_{\theta,-\alpha}=-D_{\theta, \alpha}$ 


\subsection{Case-4 : Periodic motion}

Here, the pressure gradient is $\wp(t)=p_{0} \sin w t$. The solutions are

$$
\begin{aligned}
& u=\sum_{k_{m}=1}^{\infty} \frac{2 R p_{0} w}{k_{m}}\left(\frac{I_{0}\left(k_{m}\right)-I_{0}\left(\frac{k_{m} r}{R}\right)}{I_{1}\left(k_{m}\right)}\right)\left[\frac{\cos n \alpha-\cos n \theta}{\cos n \alpha}\right] \\
& \times\left\{\frac{e^{y_{1} t}\left(y_{1}+\lambda\right)^{2}}{\left[\left(y_{1}+\lambda\right)^{2}+\lambda \beta\right]\left(y_{1}^{2}+w^{2}\right)}+\frac{e^{y_{2} t}\left(y_{2}+\lambda\right)^{2}}{\left[\left(y_{2}+\lambda\right)^{2}+\lambda \beta\right]\left(y_{2}^{2}+w^{2}\right)}\right\} \\
& +\left\{\frac{e^{i w t}}{Q_{2}^{2}}\left(\frac{I_{0}\left(Q_{2} R\right)-I_{0}\left(Q_{2} r\right)}{I_{0}\left(Q_{2} R\right)}\right)-\frac{e^{-i w t}}{Q_{3}^{2}}\left(\frac{I_{0}\left(Q_{3} R\right)-I_{0}\left(Q_{3} r\right)}{I_{0}\left(Q_{3} R\right)}\right)\right\} \\
& \times\left[\frac{p_{o}(\cos n \alpha-\cos n \theta)}{2 i \cos n \alpha}\right], \\
& +\sum_{k_{m}=1}^{\infty} \frac{2 R p_{0} w \lambda}{k_{m}}\left(\frac{I_{0}\left(k_{m}\right)-I_{0}\left(\frac{k_{m} r}{R}\right)}{I_{1}\left(k_{m}\right)}\right)\left[\frac{\cos n \alpha-\cos n \theta}{\cos n \alpha}\right] \\
& +\left\{\frac{e^{y_{1} t}\left(y_{1}+\lambda\right)}{\left[\left(y_{1}+\lambda\right)^{2}+\lambda \beta\right]\left(y_{1}^{2}+w^{2}\right)}+\frac{e^{y_{2} t}\left(y_{2}+\lambda\right)}{\left[\left(y_{2}+\lambda\right)^{2}+\lambda \beta\right]\left(y_{2}^{2}+w^{2}\right)}\right\} \\
& +\left\{\frac{e^{i w t}}{Q_{2}^{2}(\lambda+i w)}\left(\frac{I_{0}\left(Q_{2} R\right)-I_{0}\left(Q_{2} r\right)}{I_{0}\left(Q_{2} R\right)}\right)\right\}\left[\frac{p_{o} \lambda(\cos n \alpha-\cos n \theta)}{2 i \cos n \alpha}\right] \\
& -\left\{\frac{e^{-i w t}}{Q_{3}^{2}(\lambda-i w)}\left(\frac{I_{0}\left(Q_{3} R\right)-I_{0}\left(Q_{3} r\right)}{I_{0}\left(Q_{3} R\right)}\right)\right\}\left[\frac{p_{o} \lambda(\cos n \alpha-\cos n \theta)}{2 i \cos n \alpha}\right]
\end{aligned}
$$

Shear stress (Skin friction) The shear stress at the boundary at $r=R$ and $\theta= \pm \alpha$

$$
\begin{aligned}
& D_{r, R}=-2 p_{0} w\left[\frac{\cos n \alpha-\cos n \theta}{\cos n \alpha}\right] \\
& \left\{\frac{e^{y_{1} t}\left(y_{1}+\lambda\right)^{2}}{\left[\left(y_{1}+\lambda\right)^{2}+\lambda \beta\right]\left(y_{1}^{2}+w^{2}\right)}+\frac{e^{y_{2} t}\left(y_{2}+\lambda\right)^{2}}{\left[\left(y_{2}+\lambda\right)^{2}+\lambda \beta\right]\left(y_{2}^{2}+w^{2}\right)}\right\} \\
& -\left\{\frac{e^{i w t}}{Q_{2}}\left[\frac{I_{1}\left(Q_{2} R\right)}{I_{0}\left(Q_{2} R\right)}\right]+\frac{e^{-i w t}}{Q_{3}}\left[\frac{I_{1}\left(Q_{3} R\right)}{I_{0}\left(Q_{3} R\right)}\right]\right\}\left[\frac{p_{o}(\cos n \alpha-\cos n \theta)}{2 i \cos n \alpha}\right] \\
& D_{\theta, \alpha}=\sum_{k_{m}=1}^{\infty} \frac{2 R w p_{0}}{k_{m}}\left(\frac{I_{0}\left(k_{m}\right)-I_{0}\left(\frac{k_{m} r}{R}\right)}{I_{1}\left(k_{m}\right)}\right) \zeta \\
& \left\{\frac{e^{y_{1} t}\left(y_{1}+\lambda\right)}{\left[\left(y_{1}+\lambda\right)^{2}+\lambda \beta\right]\left(y_{1}^{2}+w^{2}\right)}+\frac{e^{y_{2} t}\left(y_{2}+\lambda\right)}{\left[\left(y_{2}+\lambda\right)^{2}+\lambda \beta\right]\left(y_{2}^{2}+w^{2}\right)}\right\}+\zeta \\
& \left\{\frac{p_{o} e^{i w t}}{2 i Q_{2}^{2}}\left(\frac{I_{0}\left(Q_{2} R\right)-I_{0}\left(Q_{2} r\right)}{I_{0}\left(Q_{2} R\right)}\right)-\frac{p_{0} e^{-i w t}}{2 i Q_{3}^{2}}\left(\frac{I_{0}\left(Q_{3} R\right)-I_{0}\left(Q_{3} r\right)}{I_{0}\left(Q_{3} R\right)}\right)\right\}
\end{aligned}
$$$$
D_{\theta,-\alpha}=-D_{\theta, \alpha}
$$

\section{Conclusion}

Considering that the flow takes place in a porous medium, an analytical solution for the velocity distributions for both fluid and dust in a constricted channel has been derived. Based on the solutions obtained in the form of modified Bessel functions, various plots are depicted below for different values of Hartmann number $M$, deposition thickness $\delta$ and porosity number $\eta$. It is evident from the graphs that the velocity distributions are paraboloid in nature and the flow of fluid is parallel to that of dust. It is observed that in all the four cases, the velocities of both the fluid and dust decrease as the Hartmann number increases. The increase in the porosity of the fluid however, seems have no effect on velocities of the fluid and the dust expect in the case of impulsive motion where the velocities increased with the increase in porosity factor. As expected, when the deposition thickness increases, the fluid and dust velocities increase in accord with the principle of continuity.

Further, it is observed that if the dust is very fine, i.e., mass of the dust particles is negligibly small then the relaxation time $\tau=\frac{m}{K}$ of the dust particles decreases and as $\tau \rightarrow 0$, fluid and dust velocities will be same. Also, the fluid particles reaches the steady state earlier than the dust particles. This difference is due to the fact that the time dependent pressure gradient is directly exerted on the fluid. The notations used during the above discussion are given by the following expressions

$y_{1}=\frac{-x_{1}+x_{2}}{2}, \quad y_{2}=\frac{-x_{1}-x_{2}}{2}, x_{1}=\lambda R^{2}+\beta R^{2}+\lambda_{1} R^{2}-k_{m}^{2}$,

$x_{2}=\sqrt{x_{1}^{2}-4 R^{2}\left(\lambda_{1} \lambda R^{2}+\lambda k_{m}^{2}\right)}, Q_{1}^{2}=\operatorname{Re}\left(-w-\frac{\beta s}{\lambda-w}+\lambda_{1}\right)$,

$\zeta=n \tan n \alpha$.

\section{References}

[1] P. G. Saffman, On the stability of laminar flow of a dusty gas, Journal of Fluid Mechanics, 13 (1962), 120-128.

[2] D. H. Michael and D. A. Miller, Plane parallel flow of a dusty gas, Mathematika, 13 (1966), 97-109.

[3] Brent E. Sleep, Modelling transient organic vapor transport in porous media with the dusty gas model, Advances in Water Resources, 22, 3 , (1998), 247-256.

[4] P. Mitra and P. Bhattacharyya, Unsteady hydromagnetic laminar flow of a conducting dusty fluid between two parallel plates started impulsively from rest, Acta Mechanica, 39 (1981), 171-182.

[5] K. R. Madhura and M. S. Uma, Flow of An Unsteady Conducting Dusty Fluid Through A Channel of Triangular Cross-section, International Journal of Pure and Applied Mathematical Sciences, 6 (2013), 273 298.

[6] A. J. Chamka, Unsteady flow of an electrically conducting dusty gas in a channel due to an oscillating pressure gradient, Applied Mathematical Modelling, 21 (1997), 287-292.

[7] A. J. Chamka, The Stokes Problem for a Dusty Fluid in the Presence of Magnetic Field, Heat Generation and Wall Suction Effects, International Journal of Numerical Methods for Heat and Fluid Flow, 10 (2000), 116-133.

[8] Lokenath Debnath and A. K. Ghosh, On unsteady hydromagnetic flows of a dusty Fluid between Two Oscillating Plates, Journal of Appllied Scientific Research, 45 (1988), 353-365.

[9] Nicholas Mutua, Ishmail Musyoka, Mathew Kinyanjui and Jackson Kioko, Magnetohydrodynamic Free Convention Flow of a Heat Generating Fluid past a Semi-Infinite Vertical Porous Plate with Variable Suction, International Journal of Applied Mathematical Research, 2 (2013), 345-351.

[10] C. S. Bagewadi and B. J. Gireesha, A study of two dimensional unsteady dusty fluid flow under varying pressure gradient, Tensor, N.S., 64 (2003), 232-240.

[11] K. R. Madhura, B. J. Gireesha and C. S. Bagewadi, Exact solutions of unsteady dusty fluid flow through porous media in an open rectangular channel, Advances in Theoretical and Applied Mechanics, 2 (2009), $1-7$.

[12] D. F. Young, Effect of a time-dependent stenosis on flow through a tube, Journal of Engineering and Industrial Transactions, 90 (1968), 248-254.

[13] B. E. Morgan, and D. F. Young, An integral method for the analysis of flow in arterial stenosis. Bulletin of Mathematical Biology, 36 (1974), 39-53.

[14] R. N. Pralhad and D. H. Schultz, Two-layered poiseuille flow model for blood flow through arteries of small diameter and arterioles, Biorehology, 25, 5, (1988), 715-726.

[15] R. N. Pralhad and D.H. Schultz, Modeling of arterial stenosis and its applications to blood diseases, Mathematical Biosciences, 190 (2004) 203-220.

[16] R. Ponalagusamy, Blood flow through an artery with mild stenosis: A two layered model, different shapes of stenosis and slip velocity at the wall, Journal of Applied Sciences, 7 (2007), 1071-1077.

[17] P. Chaturani and P. N. Kaloni, Two-layered poiseuille flow model for blood flow through arteries of small diameter and arterioles, Biorehol ogy, 13 (1976), 243-250.

[18] P. Chaturani and V. S. Upadhya, A two-layered model for blood flow through small diameter tubes, Biorehology, 16 (1979), 109-118.

[19] J. C. Mishra and S. Chakravarty, Flow in arteries in the presence of stenosis, Journal of Biomechanics, 19 (1986), 907-918.

[20] J. C. Mishra and B. K. Kar, Momentum integral method for studying flow characterstics of blood through a stenosed vessel, Biorheology, 26 (1989), 23-35.

[21] A. D. Patel, I. A. Salehbhai, J. Banerjee, V. K. Katiyar, A. K. Shukla, An analytical soluiton of fluid flow through narrowing systems, Italian Journal of Pure and Applied Mathematics, 29 (2012), 63-70.

[22] M. S. Uma, K. R.Madhura, Analytical solutions for a dusty fluid flow through a narrowing channel in a porous medium, Advances and Applications in Fluid Mechanics, 16 (2014), 2017-2021. 


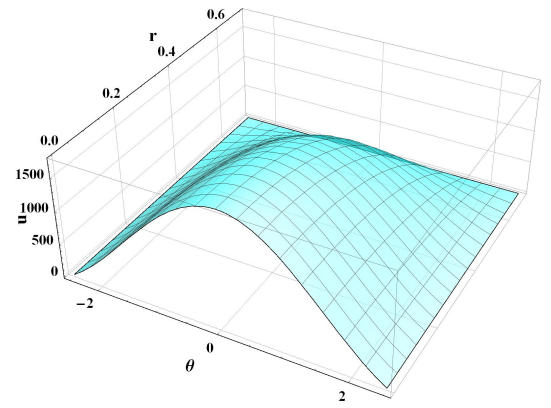

Figure 2: Variation of fluid velocity with $r$ (for $M=0.5$, Case-1)

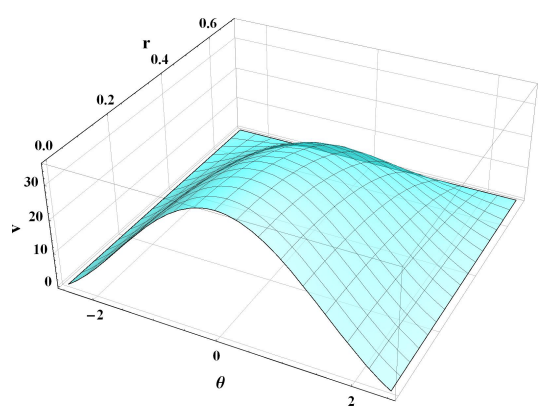

Figure 3: Variation of dust phase velocity with $r$ (for $M=0.5$, Case-1)

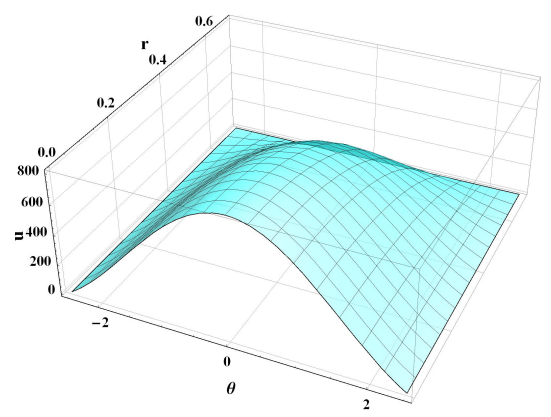

Figure 4: Variation of fluid velocity with $r$ (for $M=1$, Case-1)

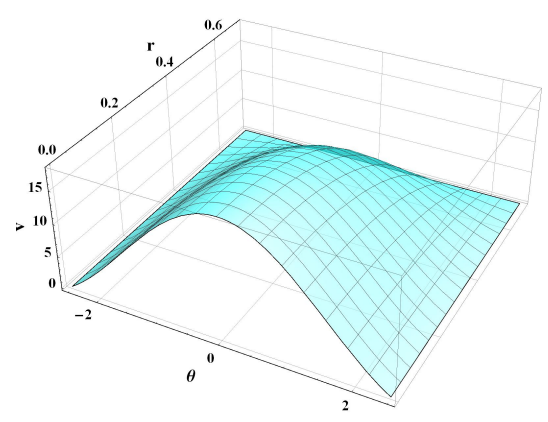

Figure 5: Variation of dust phase velocity with $r$ (for $M=1$, Case-1)

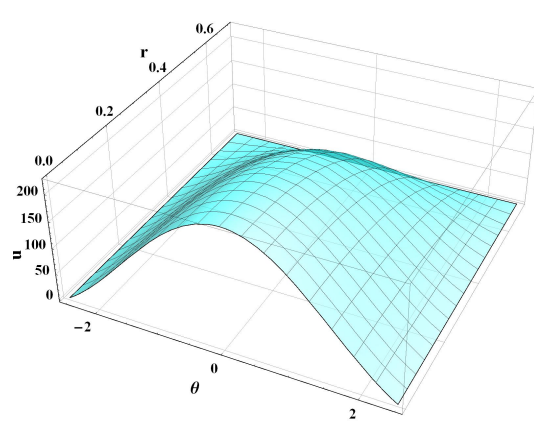

Figure 6: Variation of fluid velocity with $r$ (for $M=0.5$, Case-2)

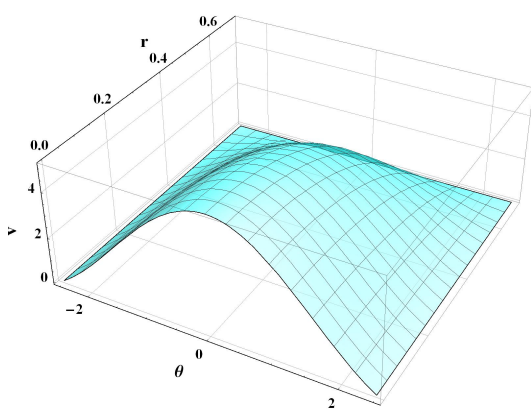

Figure 7: Variation of dust phase velocity with $r$ (for $M=0.5$, Case-2)

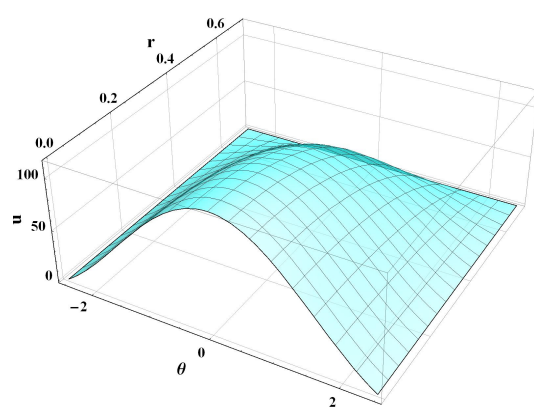

Figure 8: Variation of fluid velocity with $r$ (for $M=1$, Case-2)

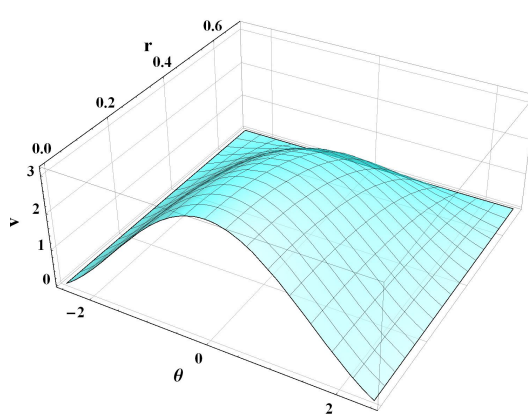

Figure 9: Variation of dust phase velocity with $r$ (for $M=1$, Case-2) 


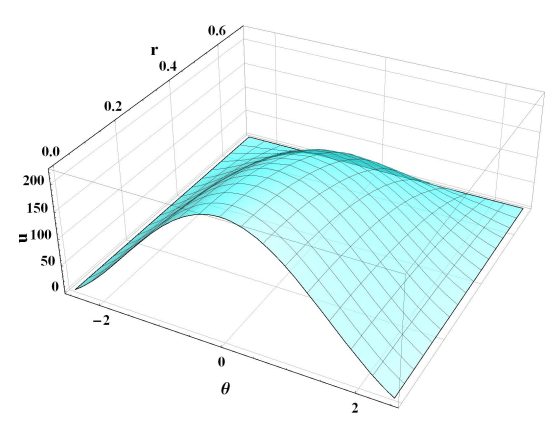

Figure 10: Variation of fluid velocity with $r$ (for $M=0.5$, Case-3)

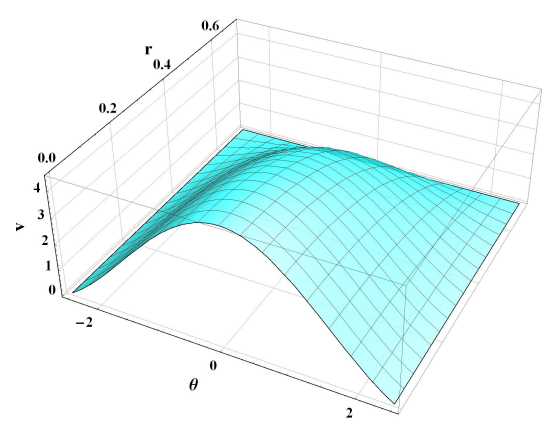

Figure 11: Variation of dust phase velocity with $r$ (for $M=0.5$, Case-3)

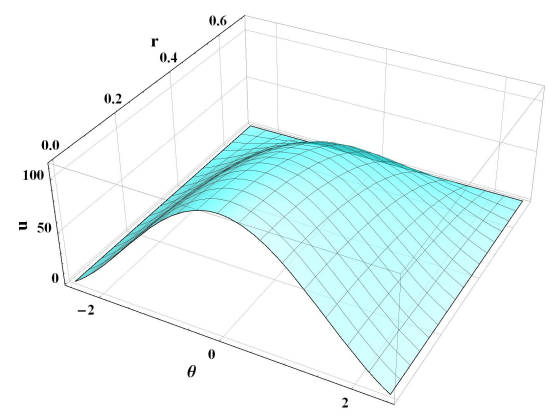

Figure 12: Variation of fluid velocity with $r$ (for $M=1$, Case-3)

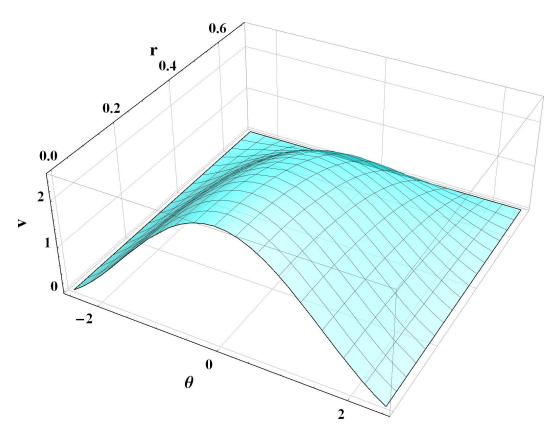

Figure 13: Variation of dust phase velocity with $r$ (for $M=1$, Case-3)

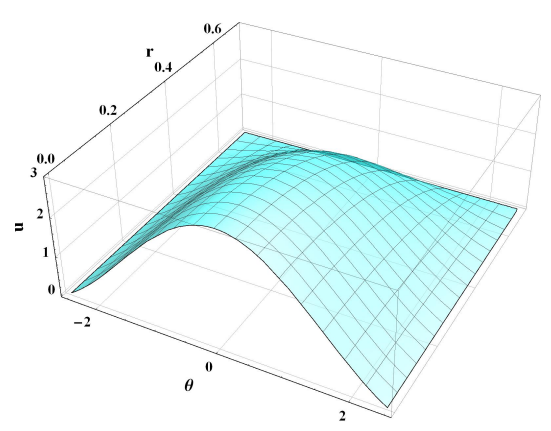

Figure 14: Variation of fluid velocity with $r$ (for $M=0.5$, Case-4)

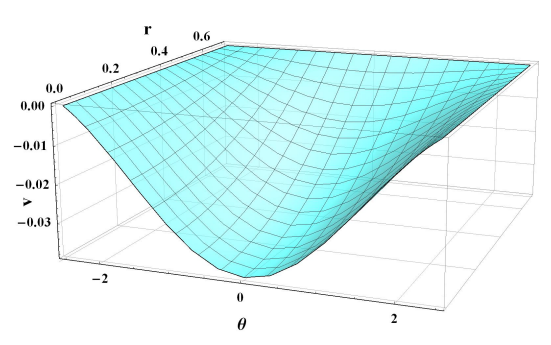

Figure 15: Variation of dust phase velocity with $r$ (for $M=0.5$, Case-4)

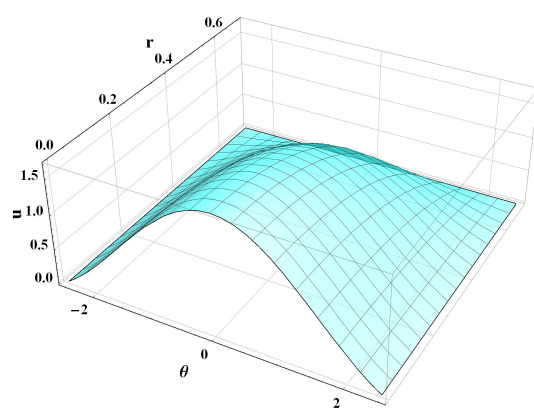

Figure 16: Variation of fluid velocity with $r$ (for $M=1$, Case-4)

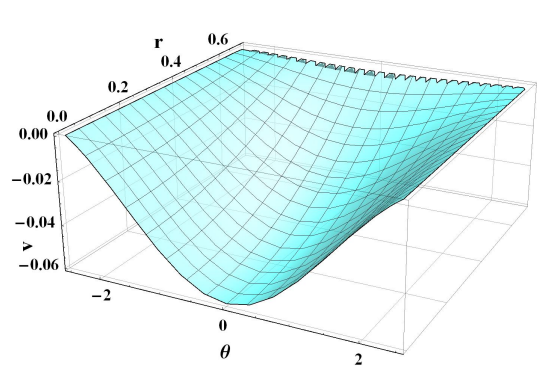

Figure 17: Variation of dust phase velocity with $r$ (for $M=1$, Case-4) 


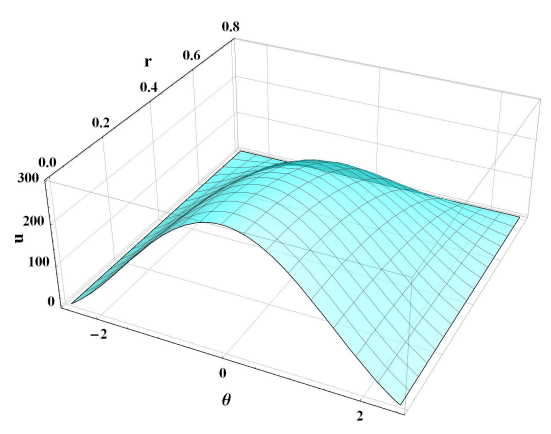

Figure 18: Variation of fluid velocity with $r$ (for $\delta=0.2$, Case-1)

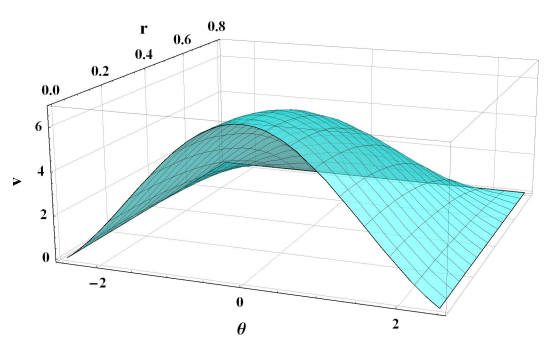

Figure 19: Variation of dust phase velocity with $r$ (for $\delta=0.2$, Case-1)

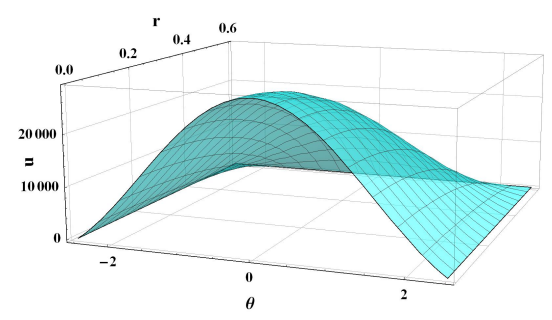

Figure 20: Variation of fluid velocity with $r$ (for $\delta=0.4$, Case-1)

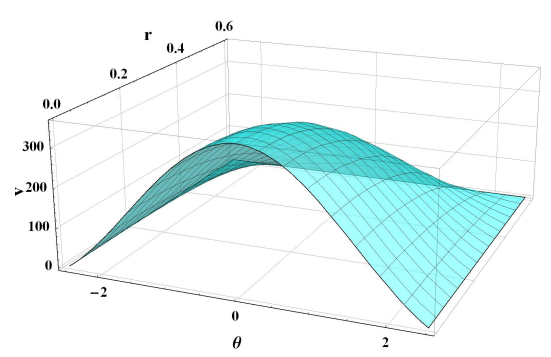

Figure 21: Variation of dust phase velocity with $r$ (for $\delta=0.4$, Case-1)

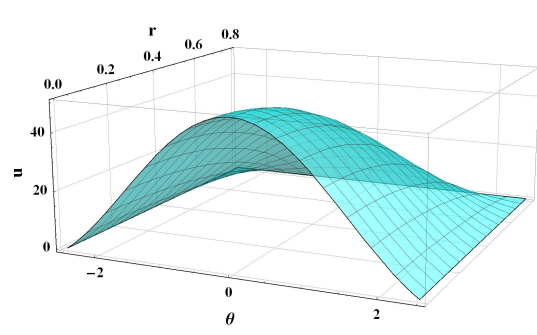

Figure 22: Variation of fluid velocity with $r$ (for $\delta=0.2$, Case-2)

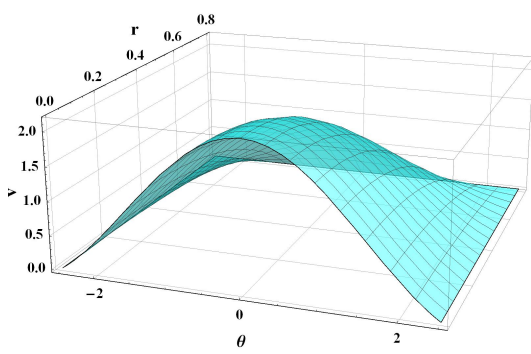

Figure 23: Variation of dust phase velocity with $r$ (for $\delta=0.2$, Case-2)

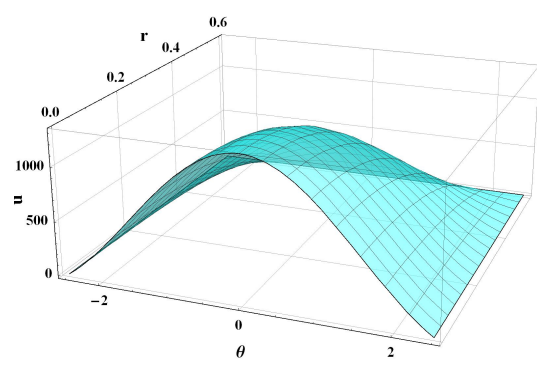

Figure 24: Variation of fluid velocity with $r$ (for $\delta=0.4$, Case-2)

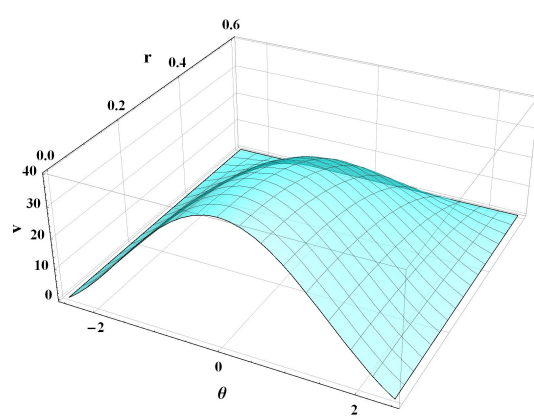

Figure 25: Variation of dust phase velocity with $r$ (for $\delta=0.4$, Case-2) 


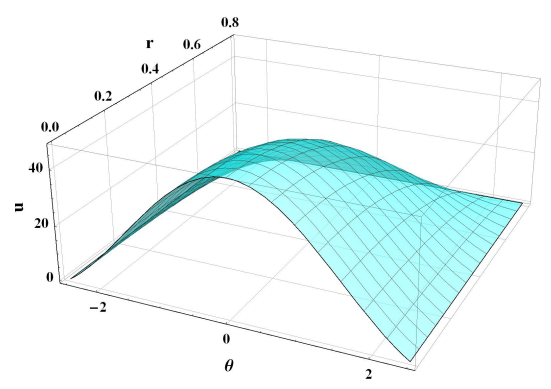

Figure 26: Variation of fluid velocity with $r$ (for $\delta=0.2$, Case-3)

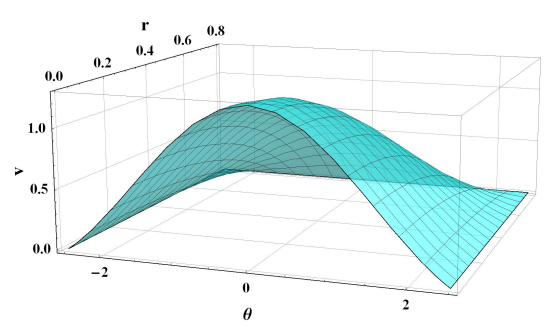

Figure 27: Variation of dust phase velocity with $r$ (for $\delta=0.2$, Case-3)

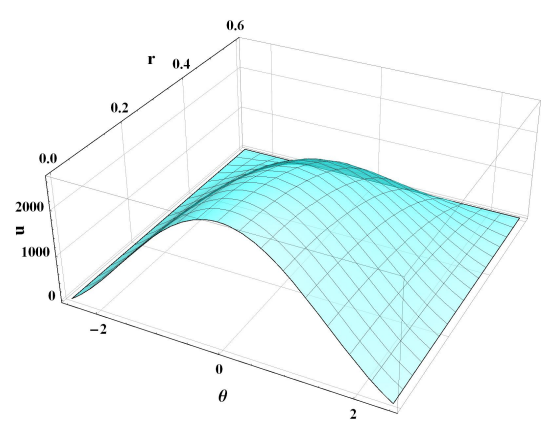

Figure 28: Variation of fluid velocity with $r$ (for $\delta=0.4$, Case-3)

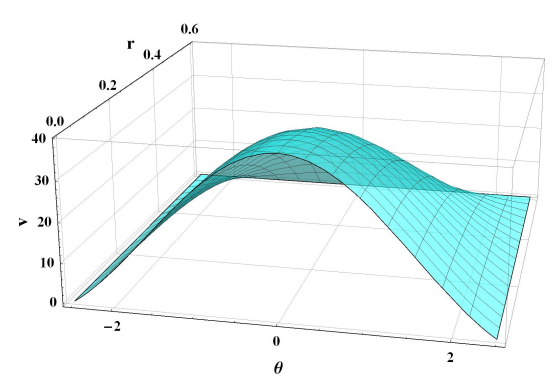

Figure 29: Variation of dust phase velocity with $r$ (for $\delta=0.4$, Case-3)

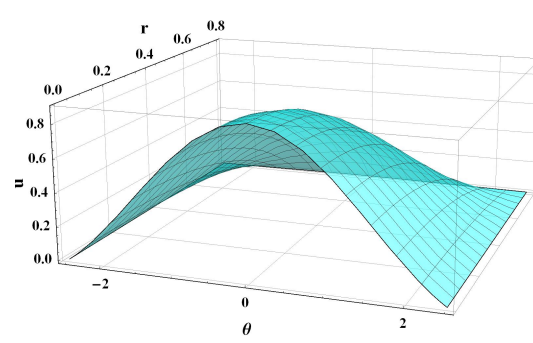

Figure 30: Variation of fluid velocity with $r$ (for $\delta=0.2$, Case-4)

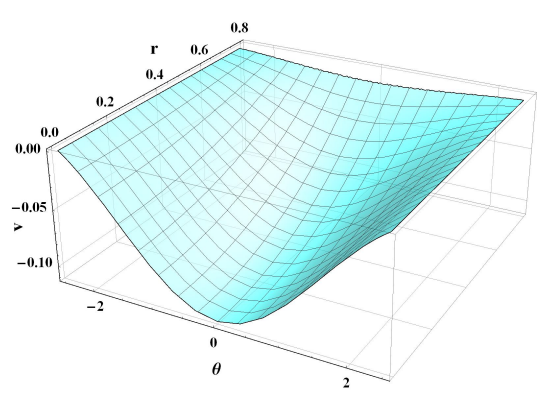

Figure 31: Variation of dust phase velocity with $r$ (for $\delta=0.2$, Case-4)

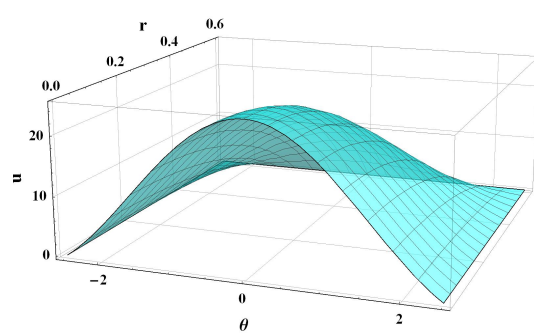

Figure 32: Variation of fluid velocity with $r$ (for $\delta=0.4$, Case-4)

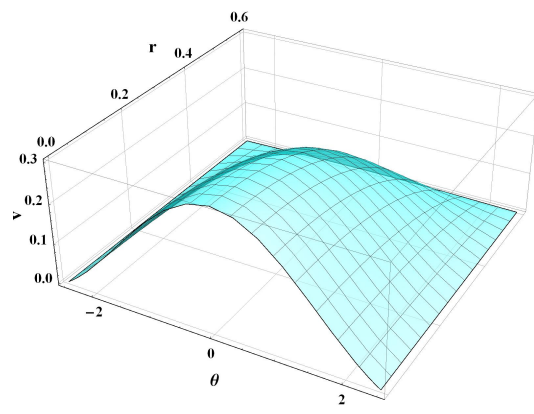

Figure 33: Variation of dust phase velocity with $r$ (for $\delta=0.4$, Case-4) 


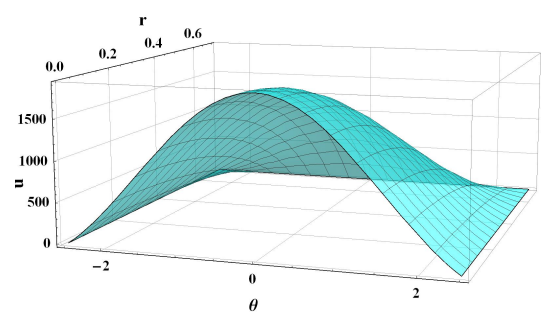

Figure 34: Variation of fluid velocity with $r$ (for $\eta=0.4$, Case-1)

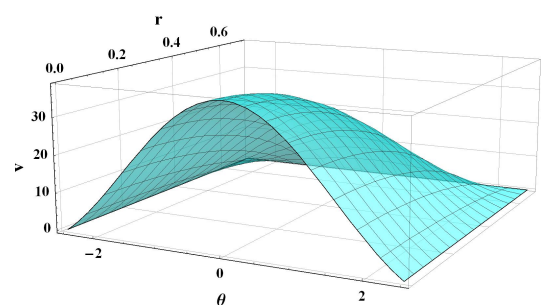

Figure 35: Variation of dust phase velocity with $r$ (for $\eta=0.4$, Case-1)

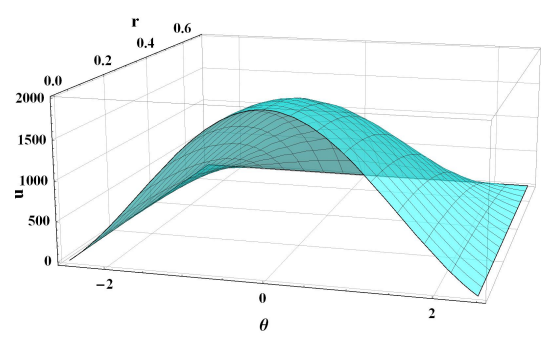

Figure 36: Variation of fluid velocity with $r$ (for $\eta=0.8$, Case-1)

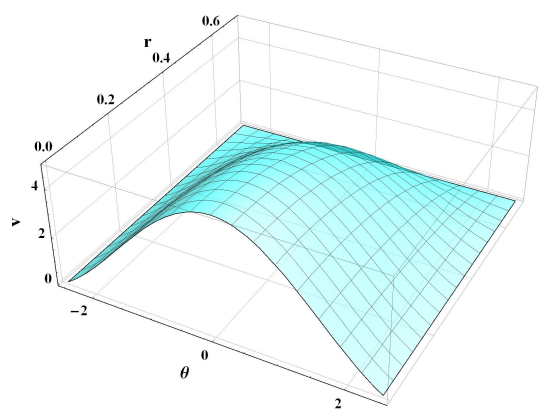

Figure 37: Variation of dust phase velocity with $r$ (for $\eta=0.8$, Case-1)

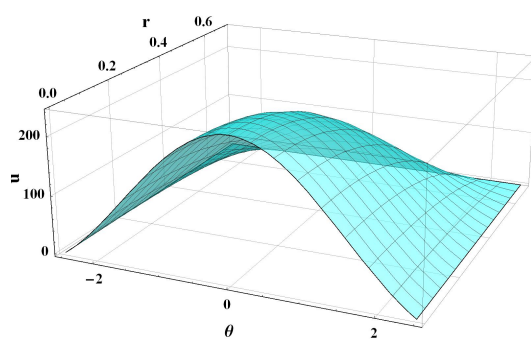

Figure 38: Variation of fluid velocity with $r$ (for $\eta=0.4$, Case-2)

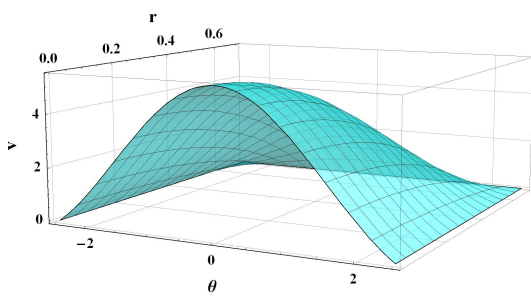

Figure 39: Variation of dust phase velocity with $r$ (for $\eta=0.4$, Case-2)

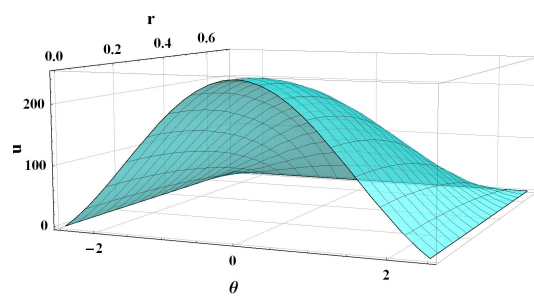

Figure 40: Variation of fluid velocity with $r$ (for $\eta=0.8$, Case-2)

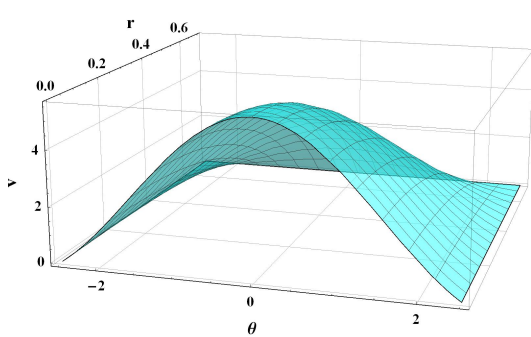

Figure 41: Variation of dust phase velocity with $r$ (for $\eta=0.8$, Case-2) 


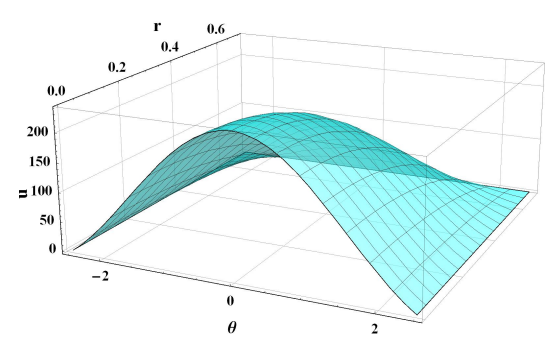

Figure 42: Variation of fluid velocity with $r$ (for $\eta=0.4$, Case-3)

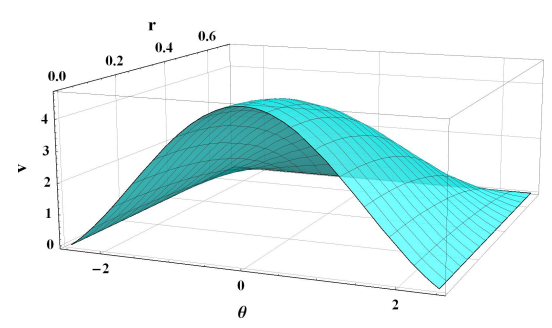

Figure 43: Variation of dust phase velocity with $r$ (for $\eta=0.4$, Case-3)

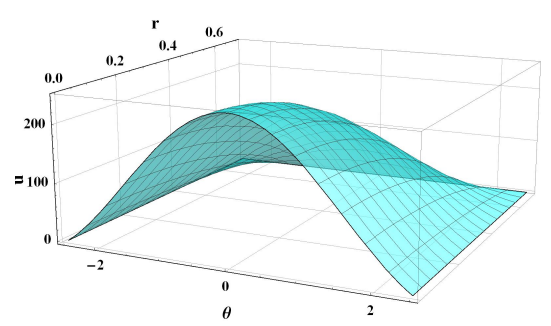

Figure 44: Variation of fluid velocity with $r$ (for $\eta=0.8$, Case-3)

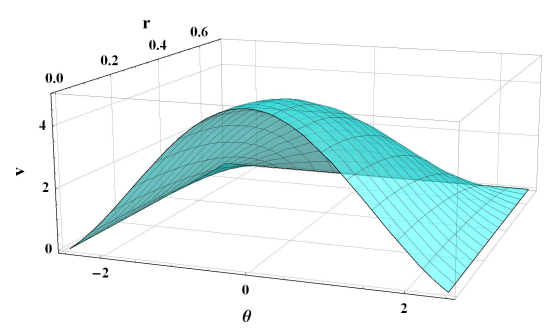

Figure 45: Variation of dust phase velocity with $r$ (for $\eta=0.8$, Case-3)

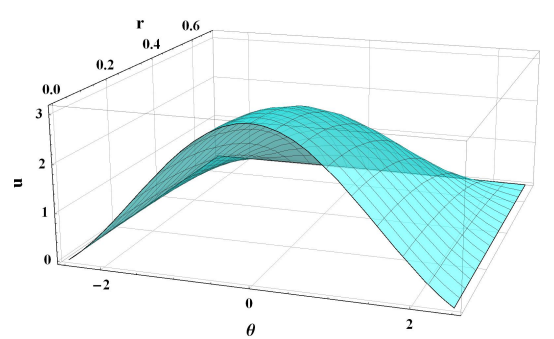

Figure 46: Variation of fluid velocity with $r$ (for $\eta=0.4$, Case-4)

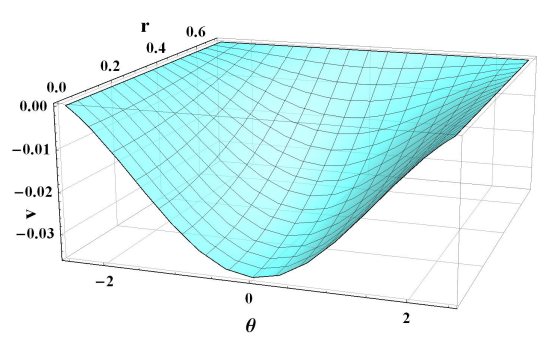

Figure 47: Variation of dust phase velocity with $r$ (for $\eta=0.4$, Case-4)

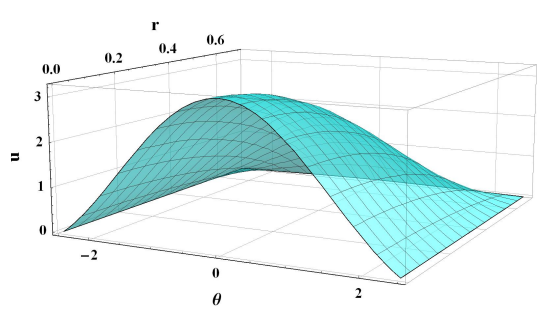

Figure 48: Variation of fluid velocity with $r$ (for $\eta=0.8$, Case-4)

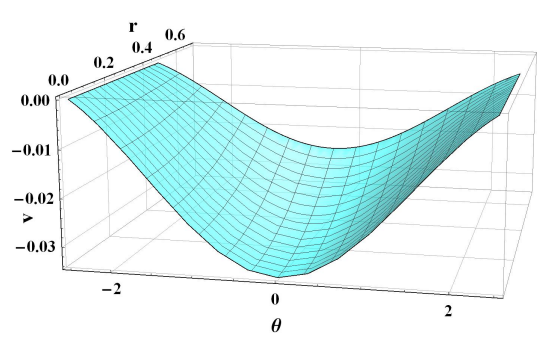

Figure 49: Variation of dust phase velocity with $r$ (for $\eta=0.8$, Case-4) 